\title{
Contracting convex hypersurfaces in Riemannian manifolds by their mean curvature
}

\section{Gerhard Huisken*}

Institut für Angewandte Mathematik, Universität Heidelberg, Im Neuenheimer Feld 294, D-6900 Heidelberg, Federal Republic of Germany

We study compact hypersurfaces $M^{n}, n \geqq 2$, without boundary, which are smoothly immersed in a Riemannian manifold $N^{n+1}$. Let $M^{n}=M_{0}$ be given locally by some diffeomorphism

$$
F_{0}: U \subset \mathbb{R}^{n} \rightarrow F_{0}(U) \subset M_{0} \subset N^{n+1} .
$$

We want to move $M_{0}$ along its mean curvature vector, that is, we want to find a whole family $F(\cdot, t)$ of diffeomorphisms corresponding to surfaces $M_{t}$, such that the evolution equation

$$
\begin{aligned}
\frac{\partial}{\partial t} F(\vec{x}, t) & =\vec{H}(\vec{x}, t) \quad \vec{x} \in U \\
F(\cdot, 0) & =F_{0}
\end{aligned}
$$

is satisfied. Here $\vec{H}(\vec{x}, t)$ is the mean curvature vector of the hypersurface $M_{t}$ at the point $F(\vec{x}, t)$ and we will see that (1) is a quasilinear parabolic system with a smooth solution at least on some short time interval. If for example $M_{0}$ is a sphere of radius $r(0)$ in $\mathbb{R}^{n+1}$, then $M_{t}$ is a family of concentric spheres of radius

$$
r(t)=\sqrt{r^{2}(0)-2 n t}
$$

which shrink towards the center of the initial sphere in finite time. It was shown in [3], that this behaviour is very typical: If the initial hypersurface $M_{0}$ $\subset \mathbb{R}^{n+1}$ is uniformly convex, then the surfaces $M_{t}$ contract smoothly to a single point in finite time and the shape of the surfaces becomes spherical at the end of the contraction.

If the ambient space $N$ is a general Riemannian manifold, the curvature of $N$ will interfere with the motion of the surfaces $M_{t}$. We want to show here that the contraction - first to a small sphere and then to a single point - is still

\footnotetext{
* This work was carried out at the Centre for Mathematical Analysis, Australian National University, Canberra
} 
working in the general case, if we only assume that the initial surface is convex enough to overcome the obstructions imposed by the geometry of $N$. By 'convex enough' we mean that the principle curvatures of $M_{0}$, i.e. the eigenvalues of the second fundamental form on $M_{0}$, are bounded from below by a positive constant depending on $N$. Since we do not have to assume a priori that the initial surface $M_{0}$ is a sphere, we also obtain results concerning the question when a locally convex hypersurface is the immersion of a sphere and under what conditions a locally convex hypersurface bounds a region diffeomorphic to a ball in $N$.

\section{The result}

In the following Latin indices range from 1 to $n$, Greek indices range from 0 to $n$ and the summation convention is understood. We denote the induced metric and the second fundamental form on $M$ by $g=\left\{g_{i j}\right\}$ and $A=\left\{h_{i j}\right\}$. The mean curvature of $M$ is the trace of the second fundamental form, $H=g^{i j} h_{i j}$. We write $\bar{R} m=\left\{\bar{R}_{\alpha \beta \gamma \delta}\right\}$ and $\bar{\nabla} \bar{R} m=\left\{\bar{\nabla}_{\sigma} \bar{R}_{\alpha \beta \gamma \delta}\right\}$ for the curvature tensor of $N$ and its covariant derivative. Let us denote by $\sigma_{x}(P)$ the sectional curvature of a 2plane $P$ at $x \in N$ and let $i_{x}(N)$ be the injectivity radius of $N$ at $x$. Let us also agree to write $T_{i j} \geqq 0$ if all eigenvalues of a symmetric tensor $T=\left\{T_{i j}\right\}$ are nonnegative.

1.1 Theorem. Let $n \geqq 2$ and $N^{n+1}$ be a smooth complete Riemannian manifold without boundary which satisfies uniform bounds

$$
\begin{gathered}
-K_{1} \leqq \sigma_{x}(P) \leqq K_{2}, \quad K_{1}, K_{2} \geqq 0 \\
|\vec{\nabla} \bar{R} m|^{2} \leqq L^{2}, \quad L \geqq 0 \\
i_{x}(N) \geqq i(N)>0 .
\end{gathered}
$$

Let $M_{0}$ be a compact connected hypersurface without boundary which is smoothly immersed in $N$, and suppose that on $M_{0}$ we have

$$
H h_{i j}>n K_{1} g_{i j}+\frac{n^{2}}{H} L g_{i j}
$$

Then (1) has a smooth solution $M_{t}$ on a finite time interval $0 \leqq t<T$ and the $M_{t}$ 's converge uniformly to a single point $0 \in N$ as $t \rightarrow T$. If we take for $t \rightarrow T$ homothetic expansions of normal coordinates around 0 such that the total area of the expanded surfaces $\tilde{M}_{t}$ is fixed, then the $\tilde{M}_{t}$ converge to a sphere of that area in the $C^{\infty}$-topology.

Remarks. (i) Inequality (2) does not depend on $K_{2}$, so positive sectional curvature in the ambient space helps toward mean curvature contraction, whereas negative sectional curvature slows it down. In particular, if $N$ is locally symmetric $(\bar{V} \bar{R} m=0)$, we have $L=0$ and condition (2) is satisfied if all eigenvalues of $A$ are bigger than $K_{1}^{1 / 2}$. If in addition the sectional curvature in the ambient 
space is non-negative, Theorem 1.1 takes exactly the same form as in Euclidean space: All locally convex hypersurfaces contract to a single point.

(ii) Condition (2) implies (for a suitable choice of normal to $M$ )

$$
H>n K_{1}^{1 / 2} \text {. }
$$

In $\S 4$ we show that (2) and (3) remain valid on $M_{t}$ for all $0 \leqq t<T$. In particular, if $N$ is locally symmetric and the initial surface $M_{0}$ is totally umbilic (i.e. $h_{i j} \equiv \frac{1}{n} H g_{i j}$ ), then this remains so and we have only to assume that (3) holds on $M_{0}$.

(iii) We will see in Lemma 7.3 that (2) and (3) are just strong enough to force all eigenvalues of the intrinsic Ricci tensor of $M_{0}$ to be positive. Thus in the two dimensional case it follows from the Gauß-Bonnet theorem that $M_{0}$ is the immersion of a sphere. In the higher dimensional case this is a consequence of Theorem 1.1: Since $M_{t}$ is a sphere for $t$ close to $T$, already $M_{0}$ must have been a sphere. We have

1.2 Corollary. Any isometric immersion $M^{n} \rightarrow N^{n+1}$, with $N$ and $M$ satisfying the conditions in Theorem 1.1, is the immersion of a sphere.

If $M_{0}$ is imbedded in $N$, then it follows from the strong parabolic maximum principle (see Lemma 3.2) that $M_{t}$ is imbedded for all $0 \leqq t<T$. Thus we have

1.3 Corollary. If $M^{n} \rightarrow N^{n+1}$ is an isometric imbedding satisfying the assumptions of Theorem 1.1, then $M$ bounds a region in $N$, and the region is diffeomorphic to a ball.

(iii) Since condition (2) remains valid for all $M_{t}, 0 \leqq t<T$, we obtain from the strong elliptic maximum principle

1.4 Corollary. If $N^{n+1}$ is a manifold with boundary $\partial N$ and the mean curvature of the boundary $H(\partial N)$ with respect to the inner normal satisfies

$$
\inf H(\partial N) \geqq-n K_{1}^{\frac{1}{2}},
$$

then $M_{t}, 0 \leqq t<T$, cannot touch $\partial N$ and all results stated above remain true.

Corollary 1.4 can be used to obtain results in manifolds $N$ without a lower bound on the injectivity radius. If for example $N$ admits an exhaustion $B_{1} \subset \subset$ $B_{2} \subset \subset B_{3} \ldots$ by compact regions $B_{l}, l \in \mathbb{N}$, such that each boundary $\partial B_{l}$ satisfies (4) with respect to the inner normal, then these boundaries act as obstacles for the evolution of $M_{0}$. Thus we have an automatic lower bound on the injectivity radius since the surfaces $M_{t}$ remain in one of the compact regions $B_{l}$ and Theorem 1.1 applies. We illustrate this with an example which also shows that inequalities (2) and (4) are optimal.

Example. Let $N=N^{3}$ be as in $([7], \S 5)$ a non-compact hyperbolic threemanifold with a finite number of ends $E_{1}, \ldots, E_{k}$ and assume that each end is homeomorphic to $T^{2} \times[0, \infty)$, where $T^{2}$ is the 2-torus. Suppose that each end 
is isometric to the quotient of a region in $\mathbb{H}^{3}$ (hyperbolic three-space in the upper half-space representation) above an interior horizontal euclidean plane by a group which is generated by two parabolic transformations which leave the point at infinity fixed. Then $L=0, K_{1}=1$ and the injectivity radius tends to zero in each end. All tori $T^{2} \times\{s\}$ are flat and all principal curvatures with respect to the inner normal are equal to -1 such that relation (4) is satisfied with equality. Thus, choosing a sequence $s_{l} \rightarrow \infty$ in each end, we can construct an exhaustion of $N$ as mentioned above and all results quoted before are true in this manifold.

The proof of Theorem 1.1 follows the proof in the euclidean case [3]. After proving in $\$ 4$ that the assumptions (2), (3) are preserved as the evolution goes on, we show in $\$ 5$ that the eigenvalues of the second fundamental form approach each other, an idea which was originally used by Hamilton, [1], for a different problem. Using this we can show that the diameter of the surfaces $M_{t}$ tends to zero at some stage and the result then follows from the assumption that the injectivity radius of $N$ is bounded from below.

\section{Preliminaries}

Let $v$ be the outer unit normal to $M_{t}$, i.e., we choose $v$ such that inequalities (2) and (3) hold with respect to $-v$ and the surfaces are moving in direction $-v$. Then for a fixed time $t$ we choose a local field of frames $e_{0}, e_{1}, \ldots, e_{n}$ in $N$ such that restricted to $M_{t}$, we have $e_{0}=v, e_{i}=\frac{\partial F}{\partial x_{i}}$. We use the same notation as in
[3] and write in particular

$$
\begin{aligned}
H & =\mathrm{g}^{i j} h_{i j}=h_{i}{ }^{i} \\
|A|^{2} & =\mathrm{g}^{i j} \mathrm{~g}^{k l} h_{i k} h_{j l}=h_{i k} h^{i k} \\
C & =\mathrm{g}^{i j} \mathrm{~g}^{k l} \mathrm{~g}^{m n} h_{i k} h_{l m} h_{n j}=h_{i k} h^{k}{ }_{l} h^{i i} \\
Z & =H \cdot C-|A|^{4} .
\end{aligned}
$$

If we mean the metric or the connection on $N$, this will be indicated by a bar, for example $\bar{g}_{\alpha \beta}, \bar{\Gamma}_{\beta \gamma}^{\alpha}$ and $\bar{\nabla}$. The Riemann curvature tensors of $M$ and $N$ will be denoted by $R m=\left\{R_{i j k l}\right\}$ and $\bar{R} m=\left\{\bar{R}_{\alpha \beta \gamma \delta}\right\}$. The relation between $A, R m$ and $\bar{R} m$ is then given by the equations of Gauß and Codazzi:

$$
\begin{gathered}
R_{i j k l}=\bar{R}_{i j k l}+h_{i k} h_{j l}-h_{i l} h_{j k} \\
\nabla_{k} h_{i j}-\nabla_{j} h_{i k}=\bar{R}_{0 i j k} .
\end{gathered}
$$

These relations now imply Simons' identity, [6], for the Laplacian of the second fundamental form on $M$. See also [5] for a simple derivation.

2.1 Lemma. We have the identities

(i) $\Delta h_{i j}=\nabla_{i} \nabla_{j} H+H h_{i l} h_{j}^{l}-|A|^{2} h_{i j}+H \bar{R}_{0 i 0 j}$

$$
-h_{i j} \bar{R}_{0 l 0}{ }^{l}+h_{j l} \bar{R}_{m i}^{l{ }^{m}+h_{i l}} \bar{R}_{m j}^{l}{ }^{m}-2 h_{l m} \bar{R}_{i}^{l m}{ }_{j}+\bar{V}_{j} \bar{R}_{0 l i}{ }^{l}+\bar{\nabla}_{l} \bar{R}_{0 i j}{ }^{l} \text {. }
$$


(ii) $\frac{1}{2} \Delta|A|^{2}=\left\langle h_{i j}, \nabla_{i} \nabla_{j} H\right\rangle+|\nabla A|^{2}+Z$

$$
\begin{aligned}
& +H h^{i j} \bar{R}_{0 i 0 j}-|A|^{2} \bar{R}_{0 l 0}{ }^{l}+2 h^{i j} h_{j l} \bar{R}_{m i}^{l m}-2 h^{i j} h^{l m} \bar{R}_{l i m j} \\
& +h^{i j}\left(\bar{\nabla}_{j} \bar{R}_{0 l i}{ }^{l}+\bar{\nabla}_{l} \bar{R}_{0 i j}{ }^{l}\right) .
\end{aligned}
$$

We also need an extension of ([3], Lemma 2.2) to hypersurfaces in general Riemannian manifolds. For that purpose we denote by $w=\left\{w_{i}\right\}$ the vector with components $w_{i}=\vec{R}_{0 l i}{ }^{l}$, i.e., $w$ is the projection of $\overline{\operatorname{Ric}}(v, \cdot)$ on $M$.

2.2 Lemma. For any $\eta>0$ we have the inequality

(i) $|\nabla A|^{2} \geqq\left(\frac{3}{n+2}-\eta\right)|\nabla H|^{2}-\frac{2}{n+2}\left(\frac{2}{n+2} \eta^{-1}-\frac{n}{n-1}\right)|w|^{2}$

and in particular

(ii) $|\nabla A|^{2}-\frac{1}{n}|\nabla H|^{2} \geqq \frac{n-1}{2 n+1}|\nabla A|^{2}-\frac{2 n}{(n-1)(2 n+1)}|w|^{2}$

$$
\geqq \frac{n-1}{2 n+1}|\nabla A|^{2}-C\left(n, K_{1}, K_{2}\right) \text {. }
$$

Proof. First note that the second inequality follows from the first one with $\eta=\frac{2(n-1)}{n(n+2)}$. To prove (i), we decompose the tensor $\nabla A=\left\{\nabla_{i} h_{j k}\right\}$ as follows:

where

$$
\nabla_{i} h_{j k}=E_{i j k}+F_{i j k}
$$

$$
\begin{aligned}
E_{i j k}= & \frac{1}{n+2}\left(\nabla_{i} H g_{j k}+\nabla_{j} H g_{i k}+\nabla_{k} H g_{i j}\right) \\
& -\frac{2}{(n+2)(n-1)} w_{i} g_{j k}+\frac{n}{(n+2)(n-1)}\left(w_{j} g_{i k}+w_{k} g_{i j}\right)
\end{aligned}
$$

Then $E_{i j k}$ has the same traces as $\nabla_{i} h_{j k}$ in view of the Codazzi equations and

Furthermore

$$
\left\langle E_{i j k}, F_{i j k}\right\rangle=0 \text {. }
$$

$$
\begin{aligned}
|E|^{2} & =\frac{3}{n+2}|\nabla H|^{2}+\frac{2 n}{(n+2)(n-1)}|w|^{2}-\frac{4}{n+2}\left\langle w_{i}, \nabla_{i} H\right\rangle \\
& \geqq\left(\frac{3}{n+2}-\eta\right)|\nabla H|^{2}+\frac{2}{n+2}\left(\frac{n}{n-1}-\frac{2}{n+2} \eta^{-1}\right)|w|^{2}
\end{aligned}
$$

which proves the Lemma. It is worth noting that in case of an Einstein manifold $N$ the vector $w$ vanishes identically and therefore $\eta$ can be chosen equal to zero.

\section{The evolution equations}

In a general Riemannian manifold $N^{n+1}$ the Gauß-Weingarten relations take the form 


$$
\begin{gathered}
\frac{\partial^{2} F^{\alpha}}{\partial x_{i} \partial x_{j}}-\Gamma_{i j}^{k} \frac{\partial F^{\alpha}}{\partial x_{k}}+\bar{\Gamma}_{\rho \sigma}^{\alpha} \frac{\partial F^{\rho}}{\partial x_{i}} \frac{\partial F^{\sigma}}{\partial x_{j}}=-h_{i j} v^{\alpha} \\
\frac{\partial v^{\alpha}}{\partial x_{j}}+\vec{\Gamma}_{\rho \sigma}^{\alpha} \frac{\partial F^{\rho}}{\partial x_{j}} v^{\alpha}=h_{j l} g^{l m} \frac{\partial F^{\alpha}}{\partial x_{m}}
\end{gathered}
$$

and evolution Eq. (1) becomes

$$
\begin{aligned}
\frac{\partial}{\partial \mathrm{t}} F^{\alpha}(\vec{x}, t) & =\vec{H}^{\alpha}(\vec{x}, t)=-H(\vec{x}, t) v(\vec{x}, t) \\
& =\Delta_{t} F^{\alpha}(\vec{x}, t)+\left\{\bar{\Gamma}_{\rho \sigma}^{\alpha} \frac{\partial F^{\rho}}{\partial x_{i}} \frac{\partial F^{\sigma}}{\partial x_{j}} g^{i j}\right\}(\vec{x}, t)
\end{aligned}
$$

where $\Delta_{t}$ is the Laplace-Beltrami operator on $M_{t}$ and the indices $\alpha, \rho, \sigma$ refer to a local coordinate system $y^{\alpha}$ in $N^{n+1}$. This is a quasi linear parabolic system and we obtain a smooth solution at least on some short time interval, cf. [1].

3.1 Lemma. If the initial surface $M_{0}$ is smooth, then (1) has a smooth solution on some maximal open time interval $0 \leqq t<T \leqq \infty$.

Since (1) is parabolic, we can also show that two surfaces moving by their mean curvature cannot overtake each other:

3.2 Lemma. (i) Let $M_{1, t}$ and $M_{2, t}$ be two smooth closed surfaces moving by their mean curvature for $0 \leqq t \leqq t_{1}$. If $M_{1}$ and $M_{2}$ are disjoint for $t=0$, they stay disjoint on the whole interval $0 \leqq t \leqq t_{1}$.

(ii) If $M_{1, t}$ is imbedded for $t=0$, then this remains so for $0 \leqq t \leqq t_{1}$.

Proof. If the surfaces were intersecting at one stage, there was a first time $0<t_{0}$ such that $M_{1, t_{0}}$ touches $M_{2, t_{0}}$ at some point $p \in N$. Let $S$ be some fixed reference surface which is tangential to the surfaces $M_{1, t_{0}}$ and $M_{2, t_{0}}$ at $p$ and assume that we have Gaussian coordinates in a neighbourhood of $S$, i.e., $y^{0}(q)$ is the length of the geodesic arc perpendicular to $S$ through $q$, and $y^{i}(q)=x_{i}(q)$ are the coordinates of the basepoint of the geodesic in $S$. Then locally around $p$ we can write $M_{1, t}$ and $M_{2, t}$ for $t \in\left(t_{0}-\varepsilon, t_{0}+\varepsilon\right)$ as graphs of functions $u_{1}(t)$ and $u_{2}(t)$ on $S$. The unit normal to $M_{i}, i=1,2$, is then given by

$$
v_{i}=\left(1+\left|\nabla u_{i}\right|^{2}\right)^{-\frac{1}{2}}\left(1,-\frac{\partial}{\partial x_{1}} u_{i}, \ldots,-\frac{\partial}{\partial x_{n}} u_{i}\right)
$$

and $u_{i}, i=1,2$, satisfies the evolution equation

$$
\frac{\partial}{\partial t} u_{i}=-\left(1+\left|\nabla u_{i}\right|^{2}\right)^{-\frac{1}{2}} \cdot H_{i}
$$

where $H_{i}$ is the mean curvature of $M_{i}$. We have $\nabla u_{1}=\nabla u_{2}=0$ at $\left(p, t_{0}\right)$ and (6) becomes a uniformly parabolic equation in a small neighbourhood of $\left(p, t_{0}\right)$. By assumption we have $u_{1}(t)>u_{2}(t)$ (say) for $t<t_{0}$ and the contradiction follows from the strong parabolic maximum principle, see for example $([4], \S \$ 3.3,3.7)$. The same argument applies for the second part of the Lemma. 
Now we want to establish evolution equations for the induced metric and the second fundamental form on $M_{r}$. It will be convenient to assume that at a fixed point $\vec{x}_{0}$ and a fixed time $t_{0}$ we have $g_{i j}\left(\vec{x}_{0}, t_{0}\right)=\delta_{i j}$ and that the coordinates $y^{\alpha}, 0 \leqq \alpha \leqq n$ for $N$ are normal coordinates at $F\left(\vec{x}_{0}, t_{0}\right)$. We can also arrange that in these coordinates $v^{\alpha}=-\delta_{0}^{\alpha}$ and $\frac{\partial F^{\alpha}}{\partial x_{i}}=\delta_{i}^{\alpha}$ at $F\left(\dot{x}_{0}, t_{0}\right)$. Then all Christoffel symbols of the connection $\bar{\Gamma}$ vanish at $F\left(\vec{x}_{0}, t_{0}\right)$ and we have only to take derivatives of the Christoffel symbols into account, which will lead to curvature terms eventually. Using the Gauß-Weingarten relations (5) and the fact that $\frac{\partial}{\partial y^{\delta}} \bar{g}_{\alpha \beta}$ vanishes at $F\left(\vec{x}_{0}, t_{0}\right)$ for $0 \leqq \delta \leqq n$ in our coordinates, we derive exactly as in ([3], Lemma 3.2 and 3.3):

3.3 Lemma. The metric and the normal of $M_{t}$ satisfy the evolution equations
(i) $\frac{\partial}{\partial t} g_{i j}=-2 H h_{i j}$
(ii) $\frac{\partial}{\partial t} v=\nabla H$.

Furthermore we have

3.4 Theorem. The second fundamental form of $M_{t}$ satisfies the evolution equation

$$
\begin{aligned}
\frac{\partial}{\partial t} h_{i j}= & \Delta h_{i j}-2 H h_{i l} h_{j}^{l}+|A|^{2} h_{i j}+h_{i j} \bar{R}_{0 l 0}{ }^{l} \\
& -h_{j l} \bar{R}_{m i}^{l m}-h_{i l} \bar{R}_{m j}^{l}{ }^{m}+2 h_{l m} \bar{R}_{i}^{l m}{ }_{j} \\
& -\bar{V}_{j} \bar{R}_{0 l i}{ }^{l}-\bar{V}_{l} \bar{R}_{0 i j}{ }^{l} .
\end{aligned}
$$

Proof. From (1) and (5) we derive

$$
\begin{aligned}
\frac{\partial}{\partial t} h_{i j}= & -\frac{\partial}{\partial t}\left(\frac{\partial^{2} F}{\partial x_{i} \partial x_{j}}, v\right)+H \bar{g}_{\alpha \beta} \frac{\partial}{\partial y^{\delta}} \bar{\Gamma}_{i \rho}^{\alpha} v^{\delta} \frac{\partial F^{\rho}}{\partial x_{j}} v^{\beta} \\
= & \left(\frac{\partial^{2}}{\partial x_{i} \partial x_{j}}(H v), v\right)-\left(\frac{\partial^{2} F}{\partial x_{i} \partial x_{j}}, \frac{\partial}{\partial x_{l}} H g^{l m} \frac{\partial F}{\partial x_{m}}\right) \\
& +H \bar{g}_{\alpha \beta} \frac{\partial}{\partial y^{\delta}} \bar{\Gamma}_{i \rho}{ }^{\alpha} v^{\delta} \frac{\partial F^{\rho}}{\partial x_{j}} v^{\beta}
\end{aligned}
$$

where we used the notation (, ) for the inner product in $N^{n+1}$. Using again (5) this is equal to

$$
\begin{aligned}
& \frac{\partial^{2}}{\partial x_{i} \partial x_{j}} H-\Gamma_{i j}^{k} \frac{\partial}{\partial x_{k}} H+H \bar{g}_{\alpha \beta} \frac{\partial}{\partial y^{\delta}} \bar{\Gamma}_{i \delta}^{\alpha} v^{\delta} \frac{\partial F}{\partial x_{j}} v^{\beta} \\
& \quad+H\left(h_{j l} g^{l m} \frac{\partial^{2} F}{\partial x_{i} \partial x_{m}}, v\right)-H \bar{g}_{\alpha \beta} \frac{\partial}{\partial x_{i}} \bar{\Gamma}_{\rho \sigma}^{\alpha} v^{\delta} \frac{\partial F^{\rho}}{\partial x_{j}} v^{\beta} \\
& =\nabla_{i} \nabla_{j} H-H h_{i l} h_{j}{ }_{j}+H \bar{R}_{0 i 0 j}
\end{aligned}
$$

and the conclusion follows from Lemma 2.1. 
From this we derive as in [3]

3.5 Corollary. We have the evolution equations

(i) $\frac{\partial}{\partial t} H=\Delta H+H\left(|A|^{2}+\overline{\operatorname{Ric}}(v, v)\right)$,

(ii) $\frac{\partial}{\partial t}|A|^{2}=\Delta|A|^{2}-2|\nabla A|^{2}+2|A|^{2}\left(|A|^{2}+\overline{\mathrm{R}}\right.$ ic $\left.(v, v)\right)$

$$
\begin{aligned}
& -4\left(h^{i j} h_{j}^{m} \bar{R}_{m l i}{ }^{l}-h^{i j} h^{l m} \bar{R}_{m i l j}\right) \\
& -2 h^{i j}\left(\bar{\nabla}_{j} \bar{R}_{0 l i}{ }^{l}+\bar{\nabla}_{l} \bar{R}_{0 i j}{ }^{l}\right)
\end{aligned}
$$

(iii) $\frac{\partial}{\partial t}\left(|A|^{2}-\frac{1}{n} H^{2}\right)=\Delta\left(|A|^{2}-\frac{1}{n} H^{2}\right)-2\left(|\nabla A|^{2}-\frac{1}{n}|\nabla H|^{2}\right)$

$$
\begin{aligned}
& +2\left(|A|^{2}-\frac{1}{n} H^{2}\right)\left(|A|^{2}+\overline{\operatorname{Ric}}(v, v)\right)-2 h^{i j}\left(\bar{\nabla}_{j} \bar{R}_{0 l i}{ }^{l}+\bar{\nabla}_{l} \bar{R}_{0 i j}{ }^{l}\right) \\
& -4\left(h^{i j} h_{j}^{m} \bar{R}_{m l i}{ }^{l}-h^{i j} h^{l m} \bar{R}_{m i l j}\right) .
\end{aligned}
$$

where $\overline{\operatorname{Ric}}(v, v)=\vec{R}_{0 l 0} l$.

Let us also note that in view of Lemma 3.3(i) the time derivative of the measure $d \mu_{t}=\mu_{t} d x$ on $M_{t}$ is the same as in the euclidean case:

$$
\frac{\partial}{\partial t} \mu_{t}=-H^{2} \mu_{t}
$$

and the area of the surfaces $M_{t}$ is decreasing very rapidly.

\section{A lower bound for the eigenvalues of $A$}

In this section we want to show that our convexity assumptions, i.e., inequalities (2) and (3) are preserved during the evolution of $M_{t}$. In view of the strict inequality in (2) there are some $\varepsilon_{1}, \varepsilon_{2}>0$ such that

$$
\begin{gathered}
H^{2} \geqq n^{2} K_{1}+n \varepsilon_{2} H^{2}, \\
H h_{i j} \geqq n K_{1} g_{i j}+\frac{n^{2}}{H} L g_{i j}+\varepsilon_{1}\left(H^{2}-n^{2} K_{1}\right) g_{i j}
\end{gathered}
$$

holds on $M_{0}$. Since $|A|^{2} \geqq \frac{1}{n} H^{2}$ and $\overline{\operatorname{Ric}}(v, v)=\bar{R}_{0 ! 0}{ }^{l} \geqq-n K_{1}$, it follows from Corollary 3.5 (i) and the maximum principle, that $(7 \mathrm{a})$ is preserved with the same $\varepsilon_{2}>0$ for all $0 \leqq t<T$. Then we have

$$
\frac{\partial}{\partial t} H \geqq \Delta H+\varepsilon_{2} H^{3}
$$


and as in ([3], Lemma 5.8) we conclude that this inequality can have a bounded solution only on a finite time interval since $\min H=H_{\min }(0)>0$. We have

4.1 Lemma. If (7a) holds on $M_{0}$, then it remains true on $M_{t}$ for $0 \leqq t<T$ and we have $T \leqq \frac{1}{2} \varepsilon_{2}^{-1} H_{\min }^{-2}(0)$.

Now we derive a lower bound for the eigenvalues of $A$.

4.2 Theorem. If for some $0<\varepsilon_{1}<\frac{1}{n}$ the inequality

$$
H h_{i j} \geqq n K_{1} g_{i j}+\frac{n^{2}}{H} L g_{i j}+\varepsilon_{1}\left(H^{2}-n^{2} K_{1}\right) g_{i j}
$$

is valid on $M_{0}$, then it remains true on $M_{t}, 0 \leqq t<T$.

Proof. We are going to show that all eigenvalues of

$$
M_{i j}=\frac{h_{i j}}{H}-\varepsilon_{1} g_{i j}-\frac{n\left(1-n \varepsilon_{1}\right)}{H^{2}} K_{1} g_{i j}-\frac{n^{2}}{H^{3}} L g_{i j}
$$

remain non-negative. First of all we need an evolution equation for $M_{i j}$. Using the evolution equation for $h_{i j}$ in Theorem 3.4 and the fact that by Corollary 3.5(i)

$$
\begin{aligned}
\frac{\partial}{\partial t} \frac{1}{H^{\alpha}}= & \Delta\left(\frac{1}{H^{\alpha}}\right)-\alpha(\alpha+1) \frac{1}{H^{\alpha+2}}|\nabla H|^{2} \\
& -\frac{\alpha}{H^{\alpha}}\left(|A|^{2}+\overline{\operatorname{Ric}}(v, v)\right) \\
= & \Delta\left(\frac{1}{H^{\alpha}}\right)+\frac{2}{H}\left\langle\nabla_{l} H, \nabla_{l}\left(\frac{1}{H^{\alpha}}\right)\right\rangle \\
& -\alpha(\alpha-1) \frac{1}{H^{\alpha+2}}|\nabla H|^{2}-\frac{\alpha}{H^{\alpha}}\left(|A|^{2}+\overline{\operatorname{Ric}}(v, v)\right),
\end{aligned}
$$

we derive as in $([3], \S 4)$ that

where

$$
\frac{\partial}{\partial t} M_{i j}=\Delta M_{i j}+\frac{2}{H}\left\langle\nabla_{l} H, \nabla_{l} M_{i j}\right\rangle+N_{i j}
$$

$$
\begin{aligned}
N_{i j}= & -2 h_{i l} h_{j}^{l}+2 \varepsilon_{1} H h_{i j}+\frac{2 n\left(1-n \varepsilon_{1}\right)}{H} K_{1} h_{i j}+\frac{2 n^{2}}{H^{2}} L h_{i j} \\
& +\frac{2 n\left(1-n \varepsilon_{1}\right)}{H^{4}} K_{1}|\nabla H|^{2} g_{i j}+\frac{6 n^{2}}{H^{5}} L|\nabla H|^{2} g_{i j} \\
& +\frac{1}{H}\left(2 h_{l m} \bar{R}_{i}^{l m}{ }_{j}-h_{j l} \bar{R}_{m i}^{l}{ }^{m}-h_{i l} \bar{R}_{m j}^{l}{ }_{m j}{ }^{m}\right)-\frac{1}{H}\left(\bar{\nabla}_{j} \bar{R}_{0 l i}{ }^{l}+\bar{\nabla}_{l} \bar{R}_{0 i j}{ }^{l}\right) \\
& +\left(\frac{2 n\left(1-n \varepsilon_{1}\right)}{H^{2}} K_{1}+\frac{3 n^{2}}{H^{3}} L\right)\left(|A|^{2}+\overline{\operatorname{Ric}}(v, v)\right) g_{i j} .
\end{aligned}
$$


In ([1], Theorem 9.1) a maximum principle for such an evolution equation was proved under the assumption that the absolute term $N_{i j}$ is a polynomial of $M_{i j}$ and $g_{i j}$. Since $\bar{R} m$ is smooth, it is easy to see that the argument is valid in our case as well. We have then only to consider the first time $t_{0}$, where at some point $p \in M_{t_{0}}$ a zero eigenvector $v=\left\{v^{i}\right\}$ of $M_{i j}$ occurs, and Theorem 4.2 is proved if we can show that $N_{i j} v^{i} v^{j}$ is non-negative. For that purpose we choose an orthonormal basis $\left(e_{1}, \ldots, e_{n}\right)$ for $T_{p} M_{t_{0}}$ such that $h_{i j}$ (and thus $M_{i j}$ ) becomes diagonal. Let us assume that $v=e_{1}$ and that $\kappa_{1}, \ldots, \kappa_{n}$ are the eigenvalues of $h_{i j}$ at $p$. Then from $M_{11}=0$ it follows that at $p$

$$
\kappa_{1}=\varepsilon H+\frac{n(1-n \varepsilon)}{H} K_{1}+\frac{n^{2}}{H^{2}} L
$$

and we obtain

$$
\begin{aligned}
N_{i j} v^{i} v^{j}= & N_{11} \geqq \frac{2}{H} \sum_{l=2}^{n} \bar{R}_{1 l 1 l}\left(\kappa_{l}-\kappa_{1}\right)-\frac{2 n}{H} L+2(1-n \varepsilon) K_{1} \\
& +\frac{3 n}{H} L-\frac{2 n^{2}(1-n \varepsilon)}{H^{2}} K_{1}^{2}-\frac{3 n^{3}}{H^{3}} L K_{1} .
\end{aligned}
$$

Here we used $|A|^{2} \geqq \frac{1}{n} H^{2}, \overline{\operatorname{Ric}}(v, v) \geqq-n K_{1}$, and $\left|\bar{\nabla}_{\alpha} \bar{R}_{\beta \gamma \delta \sigma}\right| \leqq L$. Since $\kappa_{1}$ is the smallest eigenvalue of $h_{i j}$ it follows that

$$
\begin{aligned}
& \frac{2}{H} \sum_{l=2}^{n} \vec{R}_{111 l}\left(\kappa_{l}-\kappa_{1}\right) \geqq-\frac{2}{H} K_{1} \sum_{l=2}^{n}\left(\kappa_{l}-\kappa_{1}\right) \\
& \quad=-\frac{2}{H} K_{1}\left(H-n \kappa_{1}\right)=-2 K_{1}+\frac{2 n}{H} K_{1}\left(\varepsilon_{1} H+\frac{n\left(1-n \varepsilon_{1}\right)}{H} K_{1}+\frac{n^{2}}{H^{2}} L\right) .
\end{aligned}
$$

Thus we obtain

$$
N_{i j} v^{i} v^{j} \geqq \frac{n}{H} L-\frac{n^{3}}{H^{3}} L K_{1} \geqq 0
$$

by Lemma 4.1 and the Theorem follows.

\section{The pinching estimate}

We will show that the eigenvalues of the second fundamental form come close together if the mean curvature becomes very large.

5.1 Theorem. There are constants $\delta>0$ and $C_{0}<\infty$ depending only on $M_{0}$ and the curvature bounds $K_{1}, K_{2}, L$ and $i(N)$ such that

$$
|A|^{2}-\frac{1}{n} H^{2} \leqq C_{0} \cdot H^{2-\delta}
$$

holds on $0 \leqq t<T$. 
Proof. We want to bound the function

$$
f_{\sigma}=\frac{|A|^{2}-\frac{1}{n} H^{2}}{H^{2-\sigma}}
$$

for some small $\sigma>0$. Using the evolution equations in $\S 3$ we derive similar as in [3]

5.2 Lemma. Let $\alpha=2-\sigma$. Then for any $\sigma$

$$
\begin{aligned}
\frac{\partial}{\partial t} f_{\sigma}= & \Delta f_{\sigma}+\frac{2(\alpha-1)}{H}\left\langle V_{l} H, \nabla_{l} f_{\sigma}\right\rangle \\
& -\frac{2}{H^{\alpha+2}}\left|\nabla_{i} H \cdot h_{k l}-\nabla_{i} h_{k l} \cdot H\right|^{2}-\frac{(2-\alpha)(\alpha-1)}{H^{\alpha+2}}\left(|A|^{2}-\frac{1}{n} H^{2}\right)|\nabla H|^{2} \\
& +(2-\alpha)\left(|A|^{2}+\bar{R} \operatorname{ic}(v, v)\right) f_{\sigma} \\
& -\frac{1}{H^{\alpha}}\left[4\left(h^{i j} h_{j l} \bar{R}_{m i}^{l}{ }^{m}-h^{i j} h^{l m} \bar{R}_{i l j m}\right)+h^{i j}\left(\bar{V}_{j} \bar{R}_{0 l i}^{l}+\bar{\nabla}_{l} \bar{R}_{0 i j}{ }^{l}\right)\right] .
\end{aligned}
$$

We now need the following consequences of inequality (7b) and Theorem 4.2.

5.3 Lemma. If $H>0$ and ( $7 \mathrm{~b})$ is valid with some $\varepsilon_{1}>0$, then

(i) $Z \geqq n \varepsilon_{1}^{2} H^{2}\left(|A|^{2}-\frac{1}{n} H^{2}\right)$

(ii) $\left|\nabla_{i} h_{k l} \cdot H-\nabla_{i} H h_{k l}\right|^{2} \geqq \frac{1}{4} \varepsilon_{1}^{2} H^{2}|\nabla H|^{2}-\varepsilon_{1}^{-2} c_{n} \max \left(K_{1}^{2}, K_{2}^{2}\right) H^{2}$

where $c_{n}$ here and in the following denotes a constant only depending on $n$.

Proof. This is a generalization of the result in ([3], Lemma 2.3). The proof of the first inequality carries over unchanged and to obtain the second inequality we estimate

$$
\begin{aligned}
\left|\nabla_{i} h_{k l} H-\nabla_{i} H h_{k l}\right|^{2} & \geqq \frac{1}{4}\left|\left(\nabla_{i} h_{k l}-\nabla_{k} h_{i l}\right) H-\left(\nabla_{i} H h_{k l}-\nabla_{k} H h_{i l}\right)\right|^{2} \\
& =\frac{1}{4}\left|\bar{R}_{0 l k i} H-\left(\nabla_{i} H h_{k l}-\nabla_{k} H h_{i l}\right)\right|^{2} .
\end{aligned}
$$

Rotating now the coordinates as in [3] such that $\nabla H=e_{1}|\nabla H|$, we see that this is larger than

$$
\begin{aligned}
& \frac{1}{4}\left|\bar{R}_{0221} H-\right| \nabla H\left|h_{22}\right|^{2}+\frac{1}{4}\left|\bar{R}_{0212} H+\right| \nabla H\left|h_{22}\right|^{2} \\
& \quad \geqq \frac{1}{2} \varepsilon_{1}^{2} H^{2}|\nabla H|^{2}+\frac{1}{2} H^{2} \bar{R}_{0212}^{2}+H h_{22}|\nabla H| \bar{R}_{0212} \\
& \quad \geqq \frac{1}{4} \varepsilon_{1}^{2} H^{2}|\nabla H|^{2}-\varepsilon_{1}^{-2} H^{2} \bar{R}_{0212}^{2} \\
& \quad \geqq \frac{1}{4} \varepsilon_{1}^{2} H^{2}|\nabla H|^{2}-c_{n} \varepsilon_{1}^{-2} \max \left(K_{1}^{2}, K_{2}^{2}\right) H^{2}
\end{aligned}
$$

since $h_{22} \geqq \varepsilon_{1} H$ by assumption. 
Choosing now again coordinates such that at a fixed point we have $h_{i j}$ $=\kappa_{i} \delta_{i j}$ we get (see also [5], 1.24)

$$
\begin{aligned}
h^{i j} h_{j l} \bar{R}_{m i}^{l}{ }^{m}-h^{i j} h^{l m} \bar{R}_{i l j m} & =\sum_{l<m}\left(\kappa_{l}-\kappa_{m}\right)^{2} \bar{R}_{l m l m} \\
\geqq-K_{1} \sum_{l<m}\left(\kappa_{l}-\kappa_{m}\right)^{2} & =-n K_{1}\left(|A|^{2}-\frac{1}{n} H^{2}\right) .
\end{aligned}
$$

Furthermore we have

$$
h^{i j}\left(\bar{\nabla}_{j} \bar{R}_{0 l i}{ }^{l}+\bar{\nabla}_{l} \bar{R}_{0 i j}{ }^{l}\right)=h^{i j}\left(\bar{\nabla}_{j} \bar{R}_{0 l i}{ }^{l}+\bar{\nabla}_{l} \bar{R}_{0 i j}{ }^{l}\right)
$$

where $\stackrel{h}{i j}_{i j}=h_{i j}-\frac{1}{n} H g_{i j}$ is the traceless second fundamental form. We have $\left|h_{i j}^{\circ}\right|^{2}$ $=|A|^{2}-\frac{1}{n} H^{2}$ and combining (8), (9) with Lemma 5.3, we derive from Lemma 5.2

5.4 Corollary. We have the inequality

$$
\begin{aligned}
\frac{\partial}{\partial t} f_{\sigma} \leqq & \Delta f_{\sigma}+\frac{2(\alpha-1)}{H}\left\langle\nabla_{l} H, \nabla_{l} f_{\sigma}\right\rangle-\frac{1}{2} \varepsilon_{1}^{2} \frac{1}{H^{\alpha}}|\nabla H|^{2} \\
& +\sigma|A|^{2} f_{\sigma}+C \frac{1}{H^{\alpha}}+C f_{\sigma}
\end{aligned}
$$

where $C$ only depends on $n, \varepsilon_{1}, K_{1}, K_{2}$ and $L$.

We want to exploit the negative term on the right hand side involving $|V H|^{2}$. First we conclude from Lemma 2.1 (ii) that

$$
\begin{aligned}
\frac{1}{2} \Delta|A|^{2} \geqq & \left\langle h_{i j}, \nabla_{i} \nabla_{j} H\right\rangle+Z+|\nabla A|^{2} \\
& -C H^{2}-C
\end{aligned}
$$

where $C=C\left(n, K_{1}, K_{2}, L\right)$. Then it follows that

$$
\begin{aligned}
\Delta f_{\sigma} \geqq & \frac{2}{H^{\alpha}}\left\langle\stackrel{\circ}{i j}_{i j}, \nabla_{i} V_{j} H\right\rangle+\frac{2}{H^{\alpha}} Z \\
& -\frac{2(\alpha-1)}{H}\left\langle\nabla_{l} H, \nabla_{l} f_{\sigma}\right\rangle-\frac{\alpha}{H} f_{\sigma} \Delta H \\
& -C H^{2-\alpha}-C H^{-\alpha}
\end{aligned}
$$

and we derive as in ([3], Lemma 5.4) for any $p \geqq 2, \eta>0$

$$
\begin{aligned}
& n \varepsilon_{1}^{2} \int f_{\sigma}^{p} H^{2} d \mu \leqq(2 \eta p+5) \int \frac{1}{H^{\alpha}} f_{\sigma}^{p-1}|\nabla H|^{2} d \mu \\
& \quad+\eta^{-1}(p-1) \int f_{\sigma}^{p-2}\left|\nabla f_{\sigma}\right|^{2} d \mu+c \int|\nabla H| \frac{1}{H^{\alpha}} f_{\sigma}^{p-1} d \mu \\
& \quad+C \int H^{2} f_{\sigma}^{p-1} d \mu
\end{aligned}
$$


where $C$ depends on $n, K_{1}, K_{2}, L$ and $H_{\min }^{-1}(0)$. Using now Young's inequality

we obtain

$$
x y \leqq \varepsilon x^{p}+\varepsilon^{-q / p} y^{q}, \quad \varepsilon>0, \frac{1}{p}+\frac{1}{q}=1
$$

5.5 Lemma. Let $p \geqq 2$. Then for any $\eta>0$ and any $0 \leqq \sigma \leqq \frac{1}{2}$ we have the estimate

$$
\begin{aligned}
\frac{1}{2} n \varepsilon_{1}^{2} \int f_{\sigma}^{p} H^{2} d \mu \leqq & (2 \eta p+5) \int \frac{1}{H^{\alpha}} f_{\sigma}^{p-1}|\nabla H|^{2} d \mu \\
& +\eta^{-1}(p-1) \int f_{\sigma}^{p-2}\left|\nabla f_{\sigma}\right|^{2} d \mu+C^{p}
\end{aligned}
$$

where $C$ depends on $\varepsilon_{1}, M_{0}, K_{1}, K_{2}$ and $L$.

Now we can bound $L^{p}$-norms of $f_{\sigma}$.

5.6 Lemma. There is a constant $C_{1}<\infty$ depending only on $M_{0}, K_{1}, K_{2}$ and $L$ such that for all

we have the estimate

$$
\begin{aligned}
& p \geqq 200 \varepsilon_{1}^{-2} \\
& \sigma \leqq n 2^{-5} \varepsilon_{1}^{3} p^{-\frac{1}{2}}
\end{aligned}
$$

$$
\left(\int_{M_{\mathrm{t}}} f_{\sigma}^{p} d \mu\right)^{1 / p} \leqq C_{1}, \quad 0 \leqq t<T .
$$

Proof. Using the same calculations as in ([3], Lemma 5.5) we obtain from Corollary 5.4 and Lemma 5.5 for $\sigma$ and $p$ as in (10)

$$
\frac{\partial}{\partial t} \int_{M_{t}} f_{\sigma}^{p} d \mu \leqq p \cdot C \int_{M_{t}} f_{\sigma}^{p} d \mu+p C^{p}
$$

where $C$ depends on $M_{0}, K_{1}, K_{2}$ and $L$. Thus

$$
\sup _{[0, T)} \int_{M_{t}} f_{\sigma}^{p} d \mu \leqq\left.\int f_{\sigma}^{p} d \mu\right|_{t=0}+p C^{p} \mathrm{Te}^{C T}
$$

and the conclusion follows from Lemma 4.1.

To proceed further, we need a Sobolev inequality for submanifolds of Riemannian manifolds, which was derived in [2]. In our case it takes the form

5.7 Lemma. Let $v$ be a Lipschitz function on $M$. Then

provided

$$
\left(\int_{M}|v|^{\frac{n}{n-1}} d \mu\right)^{\frac{n-1}{n}} \leqq c_{n}\left\{\int_{M}|\nabla v| d \mu+\int_{M} H|v| d \mu\right\}
$$

and

$$
K_{2}^{2}(1-\alpha)^{-\frac{2}{n}}\left(\omega_{n}^{-1}|\operatorname{supp} v|\right)^{\frac{2}{n}} \leqq 1
$$

$$
2 \rho_{0} \leqq i(N)
$$

where $\omega_{n}$ is the volume of the unit ball and

$$
\rho_{0}=K_{2}^{-1} \arcsin \left\{K_{2}(1-\alpha)^{-\frac{1}{n}}\left(\omega_{n}^{-1}|\operatorname{supp} v|\right)^{\frac{1}{n}}\right\} .
$$


Here $\alpha$ is a free parameter, $0<\alpha<1$, and

$$
c_{n}=\pi 2^{n-1} \alpha^{-1}(1-\alpha)^{-\frac{1}{n}} \frac{n}{n-1} \omega_{n}^{-\frac{1}{n}} .
$$

Now let $f_{\sigma, k}=\max \left(f_{\sigma}-k, 0\right)$ for all $k \geqq k_{0}=\sup _{M_{0}} f_{\sigma}$ and denote by $A(k)$ the set where $f_{\sigma}>k$. If we set $v=f_{\sigma, k}^{p / 2}$ for $p \geqq 200 \varepsilon_{1}^{-2}$ then we derive as in [3] from Corollary 5.4

$$
\begin{aligned}
& \frac{\partial}{\partial t} \int_{A(k)} v^{2} d \mu+\int_{A(k)}|\nabla v|^{2} d \mu \\
& \quad \leqq \sigma p \int_{A(k)} H^{2} f_{\sigma}^{p} d \mu+C_{p} \int_{A(k)} \frac{1}{H^{\alpha}} f_{\sigma, k}^{p-1} d \mu+C_{p} \int_{A(k)} f_{\sigma}^{p} d \mu \\
& \quad \leqq C_{p} \int_{A(k)} H^{2} f_{\sigma}^{D} d \mu
\end{aligned}
$$

where $C$ depends on $M_{0}, K_{1}, K_{2}$ and $L$. We have from Lemma 5.6

$$
|A(k)|=\int_{A(k)} d \mu \leqq \frac{1}{k} \int_{M} f_{\sigma} d \mu \leqq \frac{1}{k} C
$$

where $C$ depends on $C_{1}$ and $\left|M_{0}\right|$. Thus we can choose $k_{1} \geqq k_{0}$ so large that the conditions in Lemma 5.7 for $|A(k)|=|\operatorname{supp} v|$ are satisfied. Then $k_{1}$ depends on $k_{0}, i(N), M_{0}, K_{1}, K_{2}, L$ and we can now apply the Sobolev inequality as in [3] to derive a bound for $f_{\sigma}$, if $\sigma$ is small.

\section{The gradient bound}

The gradient estimate for the mean curvature in [3] is also valid in the context of Riemannian manifolds.

6.1 Theorem. For any $\eta>0$ there is a constant $C_{\eta}<\infty$ depending on $\eta, C_{0}, \delta$, $M_{0}, n, K_{1}, K_{2}$ and $L$ such that

$$
|\nabla H|^{2} \leqq \eta H^{4}+C_{\eta} .
$$

Proof. Proceeding as in ([3], Lemma 6.1) and observing that

we obtain

$$
\begin{gathered}
\Delta\left(\nabla_{k} H\right)=\nabla_{k}(\Delta H)+g^{i j} \nabla_{i} H\left(H h_{k j}-h_{k m} g^{m n} h_{n j}+\bar{R}_{k j}\right), \\
\nabla_{i}(\overline{\operatorname{Ric}}(v, v))=\bar{\nabla}_{i} \bar{R}_{0 l 0}{ }^{l}+2 \bar{R}_{m l 0}{ }^{l} h^{m}{ }_{i}
\end{gathered}
$$

6.2 Lemma. We have the evolution equation

$$
\begin{aligned}
\frac{\partial}{\partial t}|\nabla H|^{2}= & \Delta|\nabla H|^{2}-2\left|\nabla^{2} H\right|^{2}+2|A|^{2}|\nabla H|^{2} \\
& +2\left\langle\nabla_{i} H h_{m j}, \nabla_{j} H h_{i m}\right\rangle+2 H\left\langle\nabla_{i} H, \nabla_{i}|A|^{2}\right\rangle \\
& +2 \overline{\mathrm{R}} \mathrm{ic}(v, v)|\nabla H|^{2}-2 \bar{R}_{i j} \nabla^{i} H \nabla^{j} H \\
& +2 H\left\langle\bar{\nabla}_{i} R_{0 l 0}{ }^{i}, \nabla_{i} H\right\rangle+4 H\left\langle\bar{R}_{m l 0}{ }^{l} h^{m}{ }_{i}, \nabla_{i} H\right\rangle .
\end{aligned}
$$


6.3 Corollary. We have the estimate

$$
\begin{aligned}
\frac{\partial}{\partial t}|\nabla H|^{2} \leqq & \Delta|\nabla H|^{2}-2\left|\nabla^{2} H\right|^{2}+6|A|^{2}|\nabla H|^{2} \\
& +2 H\left\langle\nabla_{i} H, \nabla_{i}|A|^{2}\right\rangle+C|\nabla H|^{2}+C H^{2}
\end{aligned}
$$

where $C$ depends on $K_{1}, K_{2}$ and $L$.

6.4 Lemma. We have

$$
\begin{aligned}
& \text { (i) } \frac{\partial}{\partial t} H^{3} \geqq \Delta H^{3}-6 H|\nabla H|^{2}+3 \varepsilon_{2} H^{5} \\
& \text { (ii) } \frac{\partial}{\partial t}\left(H\left(|A|^{2}-\frac{1}{n} H^{2}\right)\right) \leqq \Delta\left(H\left(|A|^{2}-\frac{1}{n} H^{2}\right)\right)-\frac{n-1}{2 n+1} H|\nabla A|^{2} \\
& +C_{2}|\nabla A|^{2}+C_{3} H^{3}+3|A|^{2} \cdot H\left(|A|^{2}-\frac{1}{n} H^{2}\right)
\end{aligned}
$$

where $C_{2}$ and $C_{3}$ depend on $M_{0}, C_{0}, K_{1}, K_{2}, \delta$ and $L$.

Proof. (i) We have

$$
\frac{\partial}{\partial t} H^{3}=\Delta H^{3}-6 H|\nabla H|^{2}+3 H^{3}\left(|A|^{2}+\overline{\mathrm{R}} \mathrm{ic}(v, v)\right)
$$

and in view of $|A|^{2} \geqq \frac{1}{n} H^{2}$ the first inequality follows from Lemma 4.1.

(ii) From Lemma (iii) we derive

$$
\begin{gathered}
\frac{\partial}{\partial t}\left(H\left(|A|^{2}-\frac{1}{n} H^{2}\right)\right) \leqq A\left(H\left(|A|^{2}-\frac{1}{n} H^{2}\right)\right)-2\left(|\nabla A|^{2}-\frac{1}{n}|\nabla H|^{2}\right) H \\
-2\left\langle\nabla_{i} H, \nabla_{i}\left(|A|^{2}-\frac{1}{n} H^{2}\right)\right\rangle+3|A|^{2} \cdot H\left(|A|^{2}-\frac{1}{n} H^{2}\right)+C H^{3}
\end{gathered}
$$

where $C$ depends on $K_{1}, K_{2}, L$ and $H_{\min }^{-1}(0)$. Using Theorem 5.1 one estimates

$$
\begin{aligned}
& \left|\left\langle\nabla_{i} H, \nabla_{i}\left(A^{2}-\frac{1}{n} H^{2}\right)\right\rangle\right|=2\left|\left\langle\nabla_{i} H \dot{h}_{k l}^{\circ}, \nabla_{i} \stackrel{\circ}{k l}_{k l}\right\rangle\right| \\
& \quad \leqq 2|\nabla H|\left|\grave{h}_{k l}\right||\nabla A| \\
& \quad \leqq 2 n C_{0}^{\frac{1}{2}} H^{1-\delta / 2}|\nabla A|^{2} \\
& \quad \leqq \frac{n-1}{2 n+1} H|\nabla A|^{2}+C\left(n, C_{0}, \delta\right)|\nabla A|^{2}
\end{aligned}
$$

and the second inequality follows then from Lemma 2.2(ii).

Now proceeding exactly as in [3], we study the function

$$
f=\frac{|\nabla H|^{2}}{H}+P\left(|A|^{2}-\frac{1}{n} H^{2}\right) H+P C_{4}|A|^{2}-\eta H^{3}
$$


where $P$ depending only on $N$ is large and $C_{4}>0$ depends on $K_{1}, K_{2}, L$ and $C_{2}$. Using Corollary 6.3, Lemma 6.4 and Corollary 3.5(ii), we obtain as in [3]

$$
\frac{\partial}{\partial t} f \leqq \Delta f+C
$$

since all terms which do not already occur in the case $N=\mathbb{R}^{n+1}$ are of lower order. Here $C$ depends on $\eta, M_{0}, C_{0}, \delta, K_{1}, K_{2}, L$ and $\varepsilon_{2}$. This implies the estimate in Theorem 6.1.

\section{Contraction to a point}

Let again $0 \leqq t<T<\infty$ be the maximal time interval where the smooth solution of (1) exists.

7.1 Theorem. The quantity $\max _{M_{t}}|A|^{2}$ becomes unbounded as $t \rightarrow T$.

Proof. If the Lemma is false, there is some $C_{5}<\infty$ such that

$$
\max _{\boldsymbol{M}_{t}}|A|^{2} \leqq C_{5}
$$

on $0 \leqq t<T$. It follows that for $\vec{x} \in U, 0<\sigma<\rho<T$

$$
\operatorname{dist}(F(\vec{x}, \rho), F(\vec{x}, \sigma)) \leqq \int_{\sigma}^{\rho} H(\vec{x}, \tau) d \tau \leqq C(\rho-\sigma)
$$

and $F(\cdot, t)$ converges uniformly to some continuous limit function $F(\cdot, T)$. We want to show that $F(\cdot, T)$ actually represents a smooth limit surface $M_{T}$. This is then a contradiction to the maximality of $T$ in view of the local existence result in Lemma 3.1. In order to show that $F(\cdot, T)$ represents a smooth surface $M_{T}$, we have only to establish uniform bounds for all derivatives of the second fundamental form on $M_{1}, 0 \leqq t<T$, (see [3], section 8).

7.2 Lemma. If (11) holds, then for each $m \geqq 0$ there is $C_{m}<\infty$ depending on $m$, $C_{5}, M_{0}$ and $N$ such that $\max _{M_{t}}\left|\nabla^{m} A\right|^{2} \leqq C_{m}$ for all $0 \leqq t<T$.

Proof. Since $M_{t}$ stays in a compact region of $N$ in view of (12), we have $\max \left|\bar{\nabla}^{i} \bar{R} m\right| \leqq \tilde{C}_{m}$ for fixed constants $\tilde{C}_{m}$. Now, starting from the evolution $0 \leqq l \leqq m$ equation for $A$ in Theorem 3.4, one derives as in [3] and ([1], $\S 13)$ evolution equations for all iterated derivatives $\nabla^{m} A$ and obtains

$$
\begin{aligned}
\frac{\partial}{\partial t}\left|\nabla^{m} A\right|^{2} \leqq & \Delta\left|\nabla^{m} A\right|^{2}-2\left|\nabla^{m+1} A\right|^{2} \\
& +C(n, m)\left\{\sum_{i+j+k=m}\left|\nabla^{i} A\right|\left|\nabla^{j} A\right|\left|\nabla^{k} A\right|\left|\nabla^{m} A\right|\right. \\
& \left.+\tilde{C}_{m} \sum_{i \leqq m}\left|\nabla^{i} A\right|\left|\nabla^{m} A\right|+\tilde{C}_{m+1}\left|\nabla^{m} A\right|\right\} .
\end{aligned}
$$


The generalized Hölder inequality and interpolation yields

$$
\begin{aligned}
& \frac{d}{d t} \int\left|\nabla^{m} A\right|^{2} d \mu+2 \int\left|\nabla^{m+1} A\right|^{2} d \mu \\
& \quad \leqq C\left(\max _{M_{\mathrm{r}}}|A|^{2}+1\right)\left\{\int\left|\nabla^{m} A\right|^{2} d \mu+\left(\int\left|\nabla^{m} A\right|^{2} d \mu\right)^{\frac{1}{2}}\right\}
\end{aligned}
$$

where $C$ depends on $n, m$ and $\tilde{C}_{m+1}$. Then the assertion follows as in [3] from the Sobolev inequality, proving Theorem 7.1.

To proceed further, we need a lower bound for the intrinsic Ricci curvature $R_{i j}$ of the surfaces $M_{t}$.

7.3 Lemma. The intrinsic Ricci curvature $R_{i j}$ of $M_{t}$ satisfies

$$
R_{i j} \geqq(n-1) \varepsilon_{1} \varepsilon_{2} H^{2} g_{i j} .
$$

Proof. The Ricci curvature on $M$ is given by Gauß' equation

$$
R_{i j}=\bar{R}_{i l j}^{l}+H h_{i j}-h_{i l} h_{j}^{l} .
$$

Let us suppose that $R_{i j}$ is diagonal at the point of consideration, then $\bar{R}_{i l i}{ }^{l}$ is the sum of $(n-1)$ sectional curvatures and therefore larger than $-(n-1) K_{1}$.

Any eigenvalue of $H h_{i j}-h_{i l} h_{j}^{l}$ is larger than $\frac{n-1}{n} H \kappa_{1}$, where $\kappa_{1}$ is the smallest eigenvalue of $h_{i j}$. But from (2) and (7) we obtain

$$
H \kappa_{1} \geqq \varepsilon_{1}\left(n^{2} K_{1}+n \varepsilon_{2} H^{2}\right)+n K_{1}-n^{2} \varepsilon_{1} K_{1}
$$

and the conclusion follows.

Combining now Theorem 6.1, Theorem 7.1 and Lemma 7.3 exactly as in [3], we derive

\subsection{Theorem. We have $H_{\max } / H_{\min } \rightarrow 1$ as $t \rightarrow T$.}

Once this is established it follows from Theorem 7.1 that both $H_{\max }$ and $H_{\min }$ tend to infinity as $t \rightarrow T$ and therefore the diameter of $M_{t}$ tends to zero. Since the injectivity radius of $N$ is bounded from below, there is $\theta<T$ such that $M_{\theta}$ is contained in a ball $B_{\rho}(p)=\left\{q \in N \mid \operatorname{dist}_{N}(p, q)<\rho\right\}$ where $\rho$ is small compared to $i(N)$ and $\left(K_{1}+K_{2}\right)^{-1}$. It is well known that then $B_{\rho}(p)$ is a convex region. In view of the elliptic maximum principle the $M_{t}$ 's will then stay in $B_{\rho}(p)$ for all $\theta \leqq t<T$. As $H_{\min } \rightarrow \infty$ for $t \rightarrow T$, we see from Theorem 5.1 that all ratios of principal curvatures tend to one as $t \rightarrow T$. Thus for $t$ close to $T, M_{t}$ is an imbedded sphere bounding a convex region. The region enclosed by $M_{t_{2}}$ is contained in the region enclosed by $M_{t_{1}}$ for $t_{2}>t_{1} \geqq \theta$ since the surfaces are shrinking and so the $M_{t}$ 's converge to a single point as $t \rightarrow T$. The last statement of Theorem 1.1 is proved in exactly the same way as in the euclidean case ([3], $\S 10$ ), since for $t$ close to $T$ all quantities arising from the metric of $N$ are negligible compared to the mean curvature $H$ of the hypersurface. 


\section{References}

1. Hamilton, R.S.: Three-manifolds with positive Ricci curvature. J. Differ. Geom. 17, 255-306 (1982)

2. Hoffman, D., Spruck, J.: Sobolev and isoperimetric inequalities for Riemannian submanifolds. Commun. Pure. Appl. Math. 27, 715-727 (1974) and 28, 765-766 (1975)

3. Huisken, G.: Flow by mean curvature of convex surfaces into spheres. J. Differ. Geom. 20, 237$266(1984)$

4. Protter, M.H., Weinberger, H.F.: Maximum principles in differential equations. Englewood Cliffs, N.J.: Prentice Hall 1967

5. Schoen, R., Simon, L., Yau, S.T.: Curvarure estimates for minimal hypersurfaces. Acta Math. 134, $275-288$ (1975)

6. Simons, J.: Minimal varieties in Riemannian manifolds. Ann. Math. 88, 62-105 (1968)

7. Thurston, B.: The geometry and topology of three-manifolds. Notes, Princeton, 1979

Oblatum 3-XII-1984 